expression
exal
levels of p53
are enough
to suppress
CD44
expression
and limit
EGF-induced
proliferation.

TUMOUR SUPPRESSION

\section{Sharing the limelight}

The cell surface membrane protein CD44 is now an 'A-list' molecule owing to its expression on stem (and potential cancer stem) cells. However, new data from Samuel Godar, Robert Weinberg and colleagues suggest that CD44 might have to share its new-found fame with an old pro, p53.

Weinberg and colleagues had noticed that expression of CD44 in tumour tissue samples correlated with high expression levels of p53. Such levels of p53 are indicative of mutant protein expression, so they investigated a putative link between these two molecules. They established that high levels of CD44 in breast cancer samples correlated with expression of mutant (transcriptionally compromised) p53, indicating that p53 might negatively regulate CD44 expression. Using human mammary epithelial cells (BPECs) transfected with the catalytic subunit of telomerase (BPEC-T cells) to prolong their proliferation in culture, they showed that expression levels of CD44 were inversely related to p53 levels. Moreover, expression of CD44 in breast tissue from p53-null mice showed high CD44 expression levels, and restoration of $\mathrm{p} 53$ expression to this tissue resulted in decreased CD44 expression. Western blot and reverse transcriptase PCR experiments indicated that loss of p53 expression affected CD44 at the mRNA level, suggesting transcriptional control. Using a luciferase reporter vector containing the CD44 promoter sequence the authors found that p53 required a functional DNA binding domain to repress CD44 expression, and gel-shift assays and chromatin immunoprecipitation experiments showed that p53 is able to bind the CD44 promoter both in vitro and in vivo by binding to a non-canonical p53 consensus sequence.
Why does p53 need to repress CD44 expression? CD44 can promote cell growth and resistance to apoptosis, and has been shown to stimulate epidermal growth factor (EGF) signalling. Expression of short hairpin RNAs (shRNAs) that targeted either p53, CD44 or both in BPEC-T cells showed that the basal expression levels of p53 are enough to suppress CD44 expression and limit EGF-induced proliferation. In addition, ectopic expression of CD44 in these cells resulted in resistance to p53-mediated death in response to the chemotherapeutic drug doxorubicin. Therefore, $\mathrm{p} 53$ needs to suppress CD44 expression in order to limit cell proliferation and enable apoptosis. However, p53 regulates the expression of many genes that control cell-cycle progression and cell death, so how significant is this effect on CD44? Using BPLER cells, a transformed variant of BPECs that express SV40 large T antigen to inactivate $\mathrm{p} 53$ function, the authors showed that knockdown of CD44 expression reduced BPLER cell growth in mice, and increased the numbers of cells that needed to be injected to form tumours, consistent with CD44 being a marker of 'cancer stem' cells. CD44 expression can also enhance tumour progression, as suppression of p53 using shRNAs in A459 lung adenocarcinoma cells that have normal levels of p53 and CD44 resulted in increased tumour size compared with parent A459 cells and A459 cells expressing shRNAs that target both p53 and CD44.

These results indicate that CD44 is more than a mere marker of stem cells - it is able to increase cell growth and reduce cell death and will induce chemotherapy resistance when overexpressed in cells with wild-type p53. They also clearly indicate that basal expression levels of p53 have crucial functions within cells under physiological conditions, something that is often overlooked.

Nicola McCarthy Growth-inhibitory and tumour suppressive functions of $p 53$ depend on its repression of CD44 expression. Cell 134, 62-73 (2008)
ORIGINAL RESEARCH PAPER Godar, S. et al

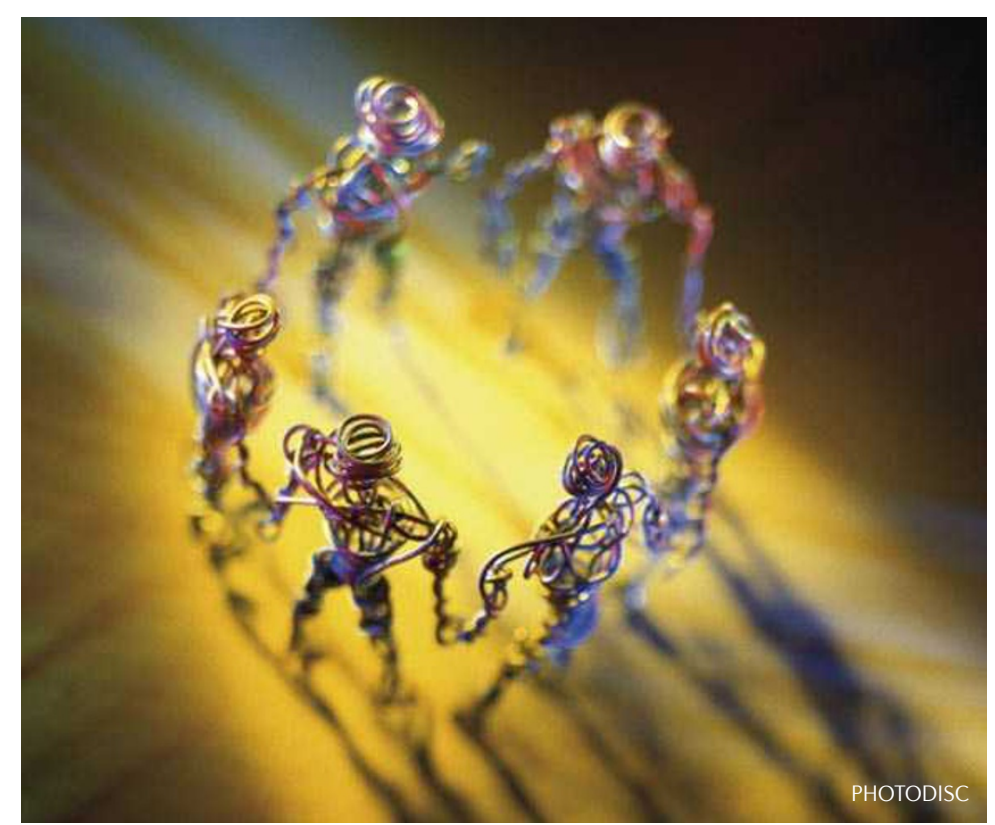

